$£$ million Balance of Payments basis seasonally adjusted

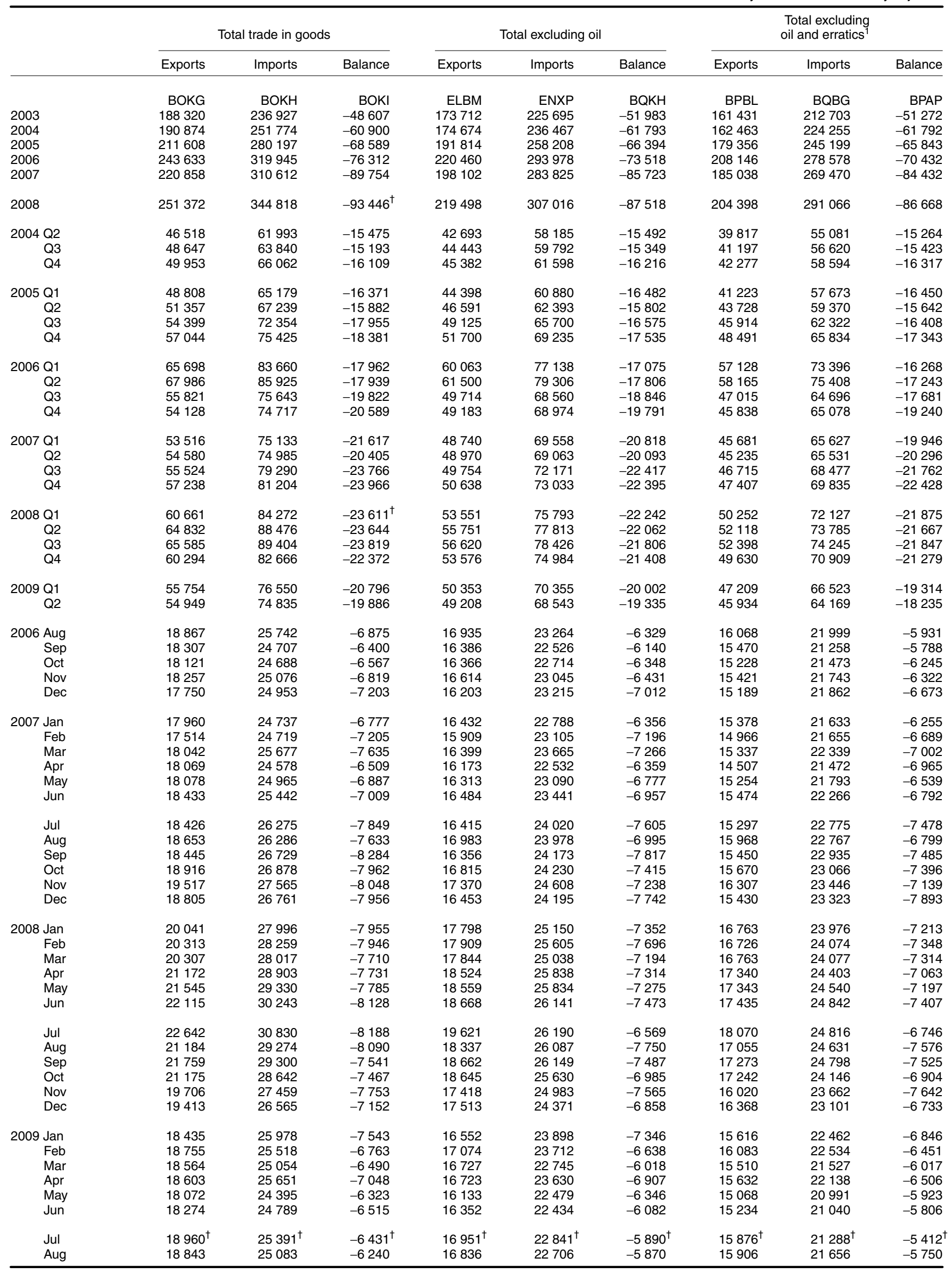


Indices 2005=100 Balance of Payments basis

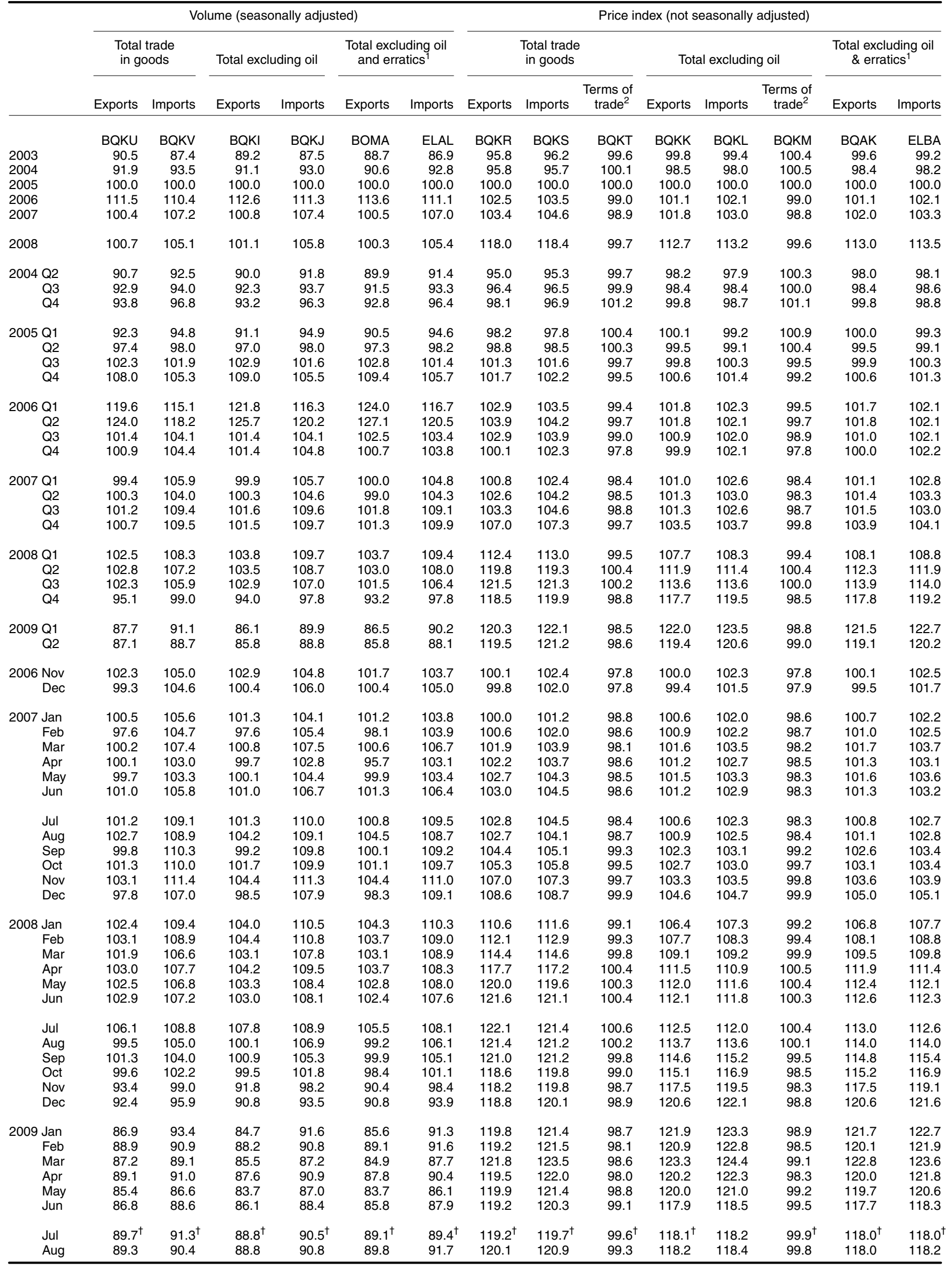


15.3 United Kingdon tratei in goods, by commoditty group'

$£$ million Balance of Payments basis seasonally adjusted

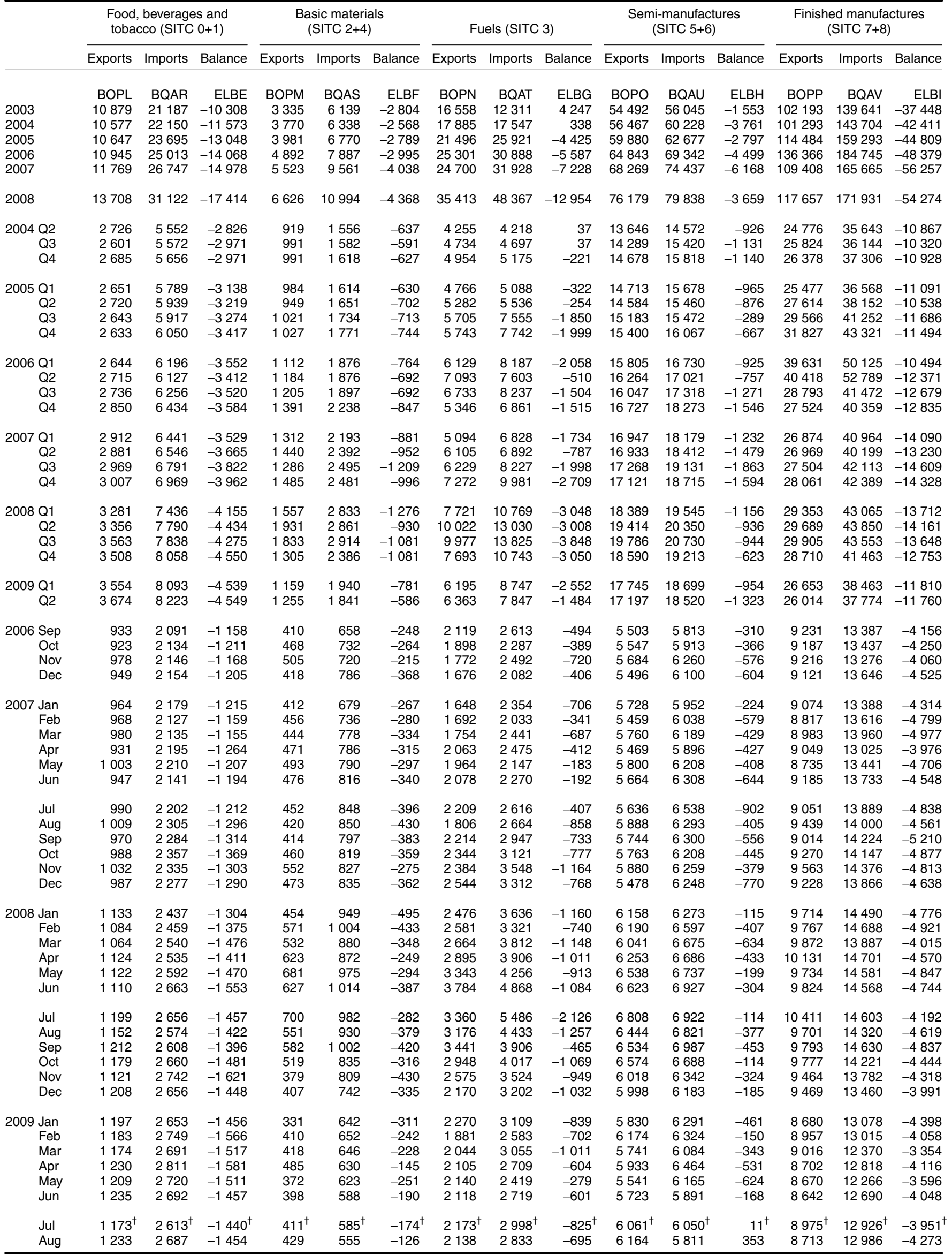

1 More commodity detail is available on a seasonally adjusted Balance of

Payments basis in tables B1 to B11 inclusive, and C1 to C4 inclusive, of the

Source: Office for National Statistics: 01633456294

Monthly Review of External Trade Statistics. 
15.4 Volume index numbers, by commodity yroup'

\begin{tabular}{|c|c|c|c|c|c|c|c|c|c|c|c|c|}
\hline & \multicolumn{2}{|c|}{$\begin{array}{c}\text { Food, beverages } \\
\text { and tobacco } \\
\text { (SITC 0+1) }\end{array}$} & \multicolumn{2}{|c|}{$\begin{array}{l}\text { Basic materials } \\
\quad(\text { SITC } 2+4)\end{array}$} & \multicolumn{2}{|c|}{ Fuels (SITC 3) } & \multicolumn{2}{|c|}{$\begin{array}{l}\text { Semi-manufactures } \\
\text { (SITC 5+6) }\end{array}$} & \multicolumn{2}{|c|}{$\begin{array}{l}\text { Finished manufactures } \\
\qquad(\text { SITC } 7+8)\end{array}$} & \multicolumn{2}{|c|}{$\begin{array}{l}\text { Total manufactures } \\
\text { (SITC } 5 \text { to } 8 \text { ) }\end{array}$} \\
\hline & Exports & Imports & Exports & Imports & Exports & Imports & Exports & Imports & Exports & Imports & Exports & Imports \\
\hline & BPEM & BQBK & BAFB & BQBL & BAFC & BQBM & BAHA & BQBN & BAHY & ELAB & BOGT & ELAJ \\
\hline 2003 & 102 & 91 & 94 & 98 & 111 & 77 & 92 & 94 & 86 & 85 & 88 & 87 \\
\hline 2004 & 101 & 97 & 98 & 98 & 103 & 95 & 96 & 100 & 87 & 90 & 90 & 93 \\
\hline 2005 & 100 & 100 & 100 & 100 & 100 & 100 & 100 & 100 & 100 & 100 & 100 & 100 \\
\hline 2006 & 101 & 104 & 114 & 108 & 101 & 101 & 107 & 105 & 117 & 115 & 113 & 112 \\
\hline 2007 & 105 & 106 & 128 & 122 & 97 & 108 & 113 & 111 & 94 & 105 & 100 & 107 \\
\hline 2008 & 109 & 106 & 135 & 120 & 99 & 110 & 114 & 109 & 92 & 102 & 100 & 104 \\
\hline 2004 Q2 & 104 & 97 & 96 & 97 & 102 & 94 & 94 & 98 & 86 & 89 & 89 & 92 \\
\hline Q3 & 99 & 98 & 102 & 98 & 103 & 95 & 97 & 102 & 88 & 90 & 91 & 93 \\
\hline Q4 & 101 & 99 & 101 & 100 & 100 & 100 & 98 & 103 & 89 & 94 & 92 & 96 \\
\hline 2005 Q1 & 100 & 99 & 98 & 99 & 104 & 95 & 98 & 101 & 87 & 92 & 90 & 95 \\
\hline Q3 & 99 & 100 & 102 & 102 & 96 & 102 & 102 & 99 & 104 & 103 & 103 & 102 \\
\hline Q4 & 98 & 102 & 101 & 101 & 96 & 107 & 103 & 100 & 113 & 108 & 110 & 106 \\
\hline 2006 Q1 & 97 & 104 & 105 & 105 & 97 & 105 & 104 & 103 & 135 & 124 & 124 & 118 \\
\hline Q2 & 100 & 102 & 110 & 107 & 108 & 95 & 107 & 104 & 139 & 131 & 128 & 123 \\
\hline Q3 & 101 & 103 & 112 & 104 & 103 & 104 & 106 & 104 & 98 & 104 & 101 & 104 \\
\hline Q4 & 105 & 106 & 129 & 118 & 95 & 99 & 112 & 110 & 95 & 102 & 101 & 104 \\
\hline 2007 Q1 & 106 & 105 & 121 & 116 & 93 & 111 & 113 & 109 & 92 & 103 & 99 & 105 \\
\hline Q2 & 104 & 106 & 131 & 121 & 101 & 99 & 113 & 110 & 92 & 101 & 100 & 104 \\
\hline Q3 & 105 & 109 & 124 & 128 & 98 & 108 & 114 & 115 & 94 & 107 & 101 & 109 \\
\hline Q4 & 104 & 107 & 136 & 121 & 95 & 116 & 111 & 111 & 95 & 107 & 101 & 108 \\
\hline 2008 Q1 & 109 & 109 & 132 & 129 & 90 & 107 & 116 & 112 & 96 & 106 & 103 & 107 \\
\hline Q2 & 108 & 108 & 147 & 125 & 99 & 102 & 117 & 112 & 94 & 105 & 102 & 107 \\
\hline Q3 & 111 & 104 & 145 & 124 & 100 & 106 & 116 & 112 & 93 & 103 & 101 & 106 \\
\hline Q4 & 106 & 101 & 115 & 103 & 108 & 123 & 104 & 100 & 86 & 94 & 92 & 95 \\
\hline 2009 Q1 & 108 & 100 & 102 & 87 & 104 & 114 & 97 & 96 & 77 & 84 & 84 & 88 \\
\hline Dec & 105 & 106 & 120 & 124 & 88 & 89 & 110 & 110 & 95 & 104 & 100 & 106 \\
\hline 2007 Jan & 106 & 107 & 115 & 109 & 92 & 123 & 115 & 107 & 93 & 102 & 101 & 103 \\
\hline Feb & 105 & 104 & 124 & 116 & 94 & 101 & 109 & 108 & 91 & 103 & 97 & 105 \\
\hline Mar & 106 & 103 & 124 & 122 & 92 & 110 & 115 & 111 & 93 & 105 & 100 & 107 \\
\hline Apr & 102 & 107 & 127 & 122 & 105 & 112 & 109 & 105 & 93 & 99 & 99 & 101 \\
\hline May & 107 & 106 & 133 & 120 & 99 & 91 & 116 & 112 & 90 & 101 & 99 & 104 \\
\hline Jun & 102 & 104 & 134 & 122 & 99 & 95 & 113 & 113 & 94 & 104 & 101 & 107 \\
\hline Jul & 106 & 107 & 126 & 132 & 103 & 102 & 112 & 118 & 94 & 106 & 100 & 109 \\
\hline Aug & 107 & 111 & 122 & 134 & 89 & 106 & 117 & 113 & 97 & 106 & 104 & 108 \\
\hline Sep & 101 & 108 & 123 & 118 & 103 & 115 & 113 & 114 & 92 & 108 & 99 & 110 \\
\hline Oct & 103 & 110 & 131 & 121 & 101 & 115 & 113 & 111 & 94 & 108 & 101 & 109 \\
\hline Nov & 108 & 107 & 151 & 120 & 93 & 120 & 115 & 112 & 97 & 110 & 103 & 110 \\
\hline Dec & 102 & 103 & 127 & 122 & 92 & 112 & 106 & 110 & 94 & 104 & 98 & 106 \\
\hline 2008 Jan & 112 & 109 & 115 & 136 & 89 & 113 & 118 & 109 & 96 & 108 & 103 & 108 \\
\hline Feb & 109 & 107 & 144 & 135 & 90 & 99 & 118 & 113 & 96 & 108 & 103 & 109 \\
\hline Mar & 106 & 110 & 138 & 117 & 90 & 109 & 113 & 113 & 97 & 103 & 102 & 105 \\
\hline Apr & 108 & 108 & 145 & 116 & 94 & 102 & 114 & 111 & 97 & 107 & 103 & 108 \\
\hline May & 108 & 108 & 147 & 126 & 99 & 100 & 118 & 111 & 93 & 105 & 102 & 107 \\
\hline Jun & 107 & 107 & 148 & 133 & 105 & 105 & 119 & 113 & 92 & 104 & 101 & 107 \\
\hline Jul & 112 & 108 & 160 & 129 & 93 & 115 & 122 & 113 & 98 & 105 & 106 & 108 \\
\hline Aug & 112 & 103 & 128 & 118 & 97 & 109 & 114 & 111 & 90 & 102 & 98 & 104 \\
\hline Sep & 110 & 102 & 147 & 124 & 109 & 93 & 113 & 111 & 91 & 103 & 99 & 105 \\
\hline Oct & 109 & 102 & 140 & 110 & 106 & 116 & 113 & 105 & 89 & 98 & 97 & 100 \\
\hline Nov & 102 & 104 & 102 & 105 & 110 & 121 & 101 & 100 & 85 & 94 & 91 & 95 \\
\hline Dec & 108 & 98 & 104 & 94 & 109 & 133 & 99 & 95 & 83 & 89 & 89 & 91 \\
\hline 2009 Jan & 108 & 100 & 81 & 85 & 113 & 126 & 95 & 97 & 76 & 86 & 82 & 89 \\
\hline Feb & 108 & 102 & 113 & 87 & 96 & 100 & 101 & 97 & 78 & 85 & 86 & 89 \\
\hline Mar & 107 & 99 & 112 & 88 & 104 & 117 & 94 & 93 & 77 & 81 & 83 & 85 \\
\hline Apr & 112 & 104 & 140 & 91 & 105 & 101 & 99 & 100 & 77 & 84 & 84 & 88 \\
\hline May & 112 & 101 & 109 & 87 & 103 & 87 & 92 & 96 & 75 & 80 & 81 & 85 \\
\hline Jun & 116 & 99 & 122 & 83 & 95 & 89 & 97 & 94 & 76 & 85 & 83 & 87 \\
\hline Jul & $112^{\dagger}$ & 98 & $118^{\dagger}$ & $80^{\dagger}$ & $99^{\dagger}$ & $104^{\dagger}$ & $102^{\dagger}$ & $96^{\dagger}$ & $78^{\dagger}$ & $86^{\dagger}$ & $86^{\dagger}$ & $89^{\dagger}$ \\
\hline Aug & 116 & 102 & 126 & 77 & 93 & 94 & 105 & 92 & 76 & 88 & 86 & 89 \\
\hline
\end{tabular}


$15.5^{\text {Price index numbers, by commodity group' }}$

\begin{tabular}{|c|c|c|c|c|c|c|c|c|c|c|c|c|}
\hline & \multicolumn{2}{|c|}{$\begin{array}{l}\text { Food, beverages and } \\
\text { tobacco }(\text { SITC } 0+1)\end{array}$} & \multicolumn{2}{|c|}{$\begin{array}{l}\text { Basic materials } \\
\quad(\text { SITC } 2+4)\end{array}$} & \multicolumn{2}{|c|}{ Fuels (SITC 3) } & \multicolumn{2}{|c|}{$\begin{array}{l}\text { Semi-manufactures } \\
\text { (SITC 5+6) }\end{array}$} & \multicolumn{2}{|c|}{$\begin{array}{l}\text { Finished manufactures } \\
\qquad(\text { SITC 7+8) }\end{array}$} & \multicolumn{2}{|c|}{$\begin{array}{l}\text { Total manufactures } \\
\text { (SITC } 5 \text { to } 8 \text { ) }\end{array}$} \\
\hline & Exports & Imports & Exports & Imports & Exports & Imports & Exports & Imports & Exports & Imports & Exports & Impor \\
\hline & BPAI & ELAN & BPAW & ELAO & BPDU & ELAP & BQAA & ELAQ & BQAB & ELAR & BQAI & \\
\hline 2003 & 100 & 98 & 87 & 93 & 64 & 60 & 99 & 94 & 102 & 103 & 100 & \\
\hline 2004 & 99 & 96 & 93 & 96 & 74 & 70 & 98 & 95 & 100 & 100 & 99 & \\
\hline 2005 & 100 & 100 & 100 & 100 & 100 & 100 & 100 & 100 & 100 & 100 & 100 & \\
\hline 2006 & 102 & 103 & 106 & 109 & 116 & 119 & 101 & 105 & 100 & 100 & 101 & \\
\hline 2007 & 106 & 108 & 110 & 118 & 119 & 122 & 102 & 107 & 101 & 100 & 101 & \\
\hline 2008 & 120 & 128 & 128 & 137 & 168 & 180 & 114 & 118 & 110 & 106 & 111 & \\
\hline
\end{tabular}

2004 Q2

Q3

98

$97 \quad 93$

$97 \quad 70$

$70 \quad 66$

2005 Q

Q2
Q4

98
99

96
97

$\begin{array}{ll}93 & 97 \\ 94 & 96 \\ 97 & 96\end{array}$

$\begin{array}{ll}80 & 76 \\ 84 & 79\end{array}$

97
98
100

94

$\begin{array}{rr}94 & 100 \\ 96 & 99 \\ 97 & 100\end{array}$

$\begin{array}{lll}100 & 99 & 98\end{array}$

100

100
100

99
100
100

$\begin{array}{rr}98 & 97 \\ 99 & 100 \\ 100 & 101\end{array}$

$\begin{array}{rr}97 & 83 \\ 100 & 93 \\ 101 & 112\end{array}$

$83 \quad 84$

$84 \quad 100$

100
99

100
101

103

103

112
112

114
111

99
100
101

99
100
100

100

100
100
100

$2006 \mathrm{Q}$

$\mathrm{Q} 2$
$\mathrm{Q} 3$
$\mathrm{Q} 4$

102
103
102
102

100
103
104

104

104
104

108
108

106
107
109

$115 \quad 119$

2007 Q1

Q2

104

Q3

$104 \quad 109$

109
113
109

113

123
122

119
126
123
107

102

101

100

100

99
100

$100 \quad 100 \quad 100$

$\begin{array}{rrr}99 & 100 & 99 \\ 100 & 100 & 100 \\ 101 & 100\end{array}$

2008 Q1

Q2

108

113

114

103

107

102
101

103
105

105
106

101

101
100

99

101

100

$101 \quad 101 \quad 102$

$100 \quad 101 \quad 102$

$\begin{array}{lll}100 & 100 & 101 \\ 100 & 100 & 101\end{array}$

Q4

$114 \quad 119$

2009 Q

Q2

121

119
125
130

110

121
118

99
114
12

102

122

139

126

101

$101 \quad 106$

$\begin{array}{ll}101 & 108 \\ 101 & 107\end{array}$

101

101
101

$100 \quad 101 \quad 102$

$100 \quad 101 \quad 102$

$\begin{array}{rrr}99 & 101 & 101 \\ 100 & 103 & 102\end{array}$

2006 Sep

Oct
Nov
Dec

122
135
134
121

132
140

156
194

166

103

107

103

$\begin{array}{lll}103 & 107 & 106 \\ 105 & 110 & 108\end{array}$

106
109

109
111
115

$\begin{array}{lll}105 & 110 & 108 \\ 106 & 112 & 110\end{array}$

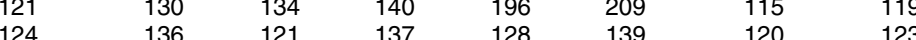

$\begin{array}{lllllll}140 & 122 & 136 & 106 & 123 & 126 & 126\end{array}$

115

113

117

117

$126 \quad 138$

122

136
131

106
121

123
136

126
121

119
118

115

121

$100 \quad 100 \quad 101$

$\begin{array}{ll}101 & 103 \\ 102 & 104\end{array}$

104
104

107
107
106

110
113

$112 \quad 111$

Dec

102
102

103

105

113
112

103
102

106

$101 \quad 106$

$101 \quad 106$

2007 Jan

Feb

102

103

Mar

May

105
104
105

Jun

105

104
106

106
106

106

106
108
112

112

112
113

112
113
117

$\begin{array}{ll}113 & 120 \\ 113 & 122\end{array}$

95

95
98
105

109

100

100

99

99
98

100

100
99

$\begin{array}{ll}100 & 101 \\ 100 & 102\end{array}$

Jul

Sep

Sep

Nov

$105 \quad 106$

112

120

113

95

101
109

115
115

100

101
102

101

102

105

105
105
107

107

108

100

101
101

101

101

$100 \quad 100 \quad 101$

$100 \quad 101 \quad 101$

$\begin{array}{rrr}101 & 101 & 103 \\ 99 & 101 & 102\end{array}$

$\begin{array}{lll}100 & 101 & 102\end{array}$

$\begin{array}{lll}100 & 101 & 102\end{array}$

2008 Ja

Jan

Feb

Apr

May
Jun

$105 \quad 106$

$\begin{array}{llll}109 & 117 & 123 & 128\end{array}$

100

107

$109-117$

119

122

$\begin{array}{llll}110 & 119 & 123 & 128\end{array}$

$\begin{array}{llllll}107 & 111 & 109 & 120 & 129 & 136\end{array}$

$\begin{array}{llll}108 & 113 & 109 & 122 \\ 110 & 115 & 113 & 123\end{array}$

142
147

148
152

101
102

102

102

103
104

107
107

107
107

$113 \quad 117$

$117 \quad 119$

$119 \quad 129$

$\begin{array}{llll}114 & 120 & 121 & 132\end{array}$

$\begin{array}{ll}116 & 121 \\ 119 & 123\end{array}$

$120-123-133$

$133 \quad 139$

$150 \quad 160$

$\begin{array}{ll}150 & 160 \\ 154 & 164 \\ 164 & 174\end{array}$

$\begin{array}{lll}139 & 176 & 174 \\ 141 & 196 & 207\end{array}$

$\begin{array}{llll}136 & 141 & 196 & 207\end{array}$

Jul 122

$$
\text { Aug }
$$

Sep

Oct

$120 \quad 129$

210

223

106
107

107
109

109
112

112

113

100

100
102

102

102
102

108

104

$\begin{array}{rll}99 & 100 & 101 \\ 99 & 100 & 101 \\ 100 & 102 & 102\end{array}$

$\begin{array}{lll}100 & 102 & 102 \\ 99 & 102 & 101\end{array}$

$\begin{array}{lll}99 & 102 & 101\end{array}$

$\begin{array}{rrr}99 & 103 & 102 \\ 101 & 104 & 103\end{array}$

$$
\text { Dec }
$$

$121 \quad 132$

$\begin{array}{ll}121 & 134 \\ 124 & 136\end{array}$

$\begin{array}{ll}137 & 139 \\ 135 & 139 \\ 131 & 142 \\ 121 & 136 \\ 120 & 137\end{array}$

2009 Jan

Jan

Feb

Mar

May
Jun

$127 \quad 139$

123

$139 \quad 212 \quad 226$

113

$\begin{array}{ll}194 & 209 \\ 181 & 193\end{array}$

$181 \quad 193$

136
137

127

162
139

115
117

116

120
124

110

112
114

116

117

105

107
107

109 


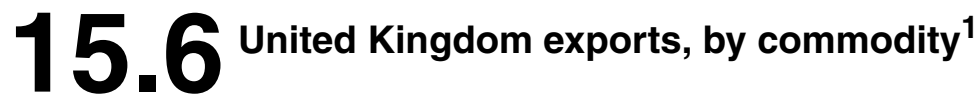

$£$ million Balance of Payments-consistent basis seasonally adjusted

\begin{tabular}{|c|c|c|c|c|c|c|c|c|c|c|c|c|}
\hline & & 2007 & 2008 & $\begin{array}{r}2008 \\
\text { Q4 }\end{array}$ & $\begin{array}{r}2009 \\
\text { Q1 }\end{array}$ & $\begin{array}{r}2009 \\
\text { Q2 }\end{array}$ & $\begin{array}{r}2009 \\
\text { Mar }\end{array}$ & $\begin{array}{r}2009 \\
\text { Apr }\end{array}$ & $\begin{array}{r}2009 \\
\text { May }\end{array}$ & $\begin{array}{r}2009 \\
\text { Jun }\end{array}$ & $\begin{array}{r}2009 \\
\text { Jul }\end{array}$ & $\begin{array}{r}2009 \\
\text { Aug }\end{array}$ \\
\hline 0. Food and live animals & BOGG & 7374 & 8681 & 2273 & 2318 & 2336 & 775 & 784 & 770 & 782 & $746^{\dagger}$ & \\
\hline \multicolumn{13}{|l|}{ Of which: } \\
\hline 01. Meat and meat preparations & BOGS & 839 & 1165 & 324 & 310 & 318 & 104 & 107 & 106 & 105 & $97^{\dagger}$ & \\
\hline 02. Dairy products and eggs & BQMS & 807 & 885 & 212 & 207 & 205 & 70 & 74 & 60 & 71 & $66^{\dagger}$ & \\
\hline $04 \& 08$. Cereals and animal feeding stuffs & BQMT & 1791 & 2283 & 625 & 617 & 624 & 209 & 207 & 205 & 212 & $198^{\dagger}$ & \\
\hline 1. Beverages and tobacco & BQMZ & 4395 & 5027 & 1235 & 1236 & 1338 & 399 & 446 & 439 & 453 & $427^{\dagger}$ & \\
\hline 11. Beverages & BQNB & 4093 & 4580 & 1118 & 1126 & 1231 & 362 & 408 & 404 & 419 & $397^{\dagger}$ & \\
\hline 12. Tobacco & BQOW & 302 & 447 & 117 & 110 & 107 & 37 & 38 & 35 & 34 & $30^{\dagger}$ & \\
\hline 2. Crude materials & $\mathrm{BQOX}$ & 5196 & 6266 & 1215 & 1017 & 1157 & 373 & 440 & 344 & 373 & $386^{\dagger}$ & \\
\hline
\end{tabular}

Of which:

24. Wood, lumber and cork
25. Pulp and waste paper
26. Textile fibres
28. Metal ores

3. Fuels

33. Petroleum and petroleum products

32, 34 and 35 . Coal, gas and electricity

\section{Animal and vegetable oils and fats}

5. Chemicals

Of which:

$$
\begin{aligned}
& \text { 51. Organic chemicals } \\
& \text { 52. Inorganic chemicals } \\
& \text { 53. Colouring materials } \\
& \text { 54. Medicinal products } \\
& \text { 55. Toilet preparations } \\
& \text { 57 \& 58. Plastics }
\end{aligned}
$$

6. Manufactures classified chiefly by material

Of which:

$$
\begin{aligned}
& \text { 63. Wood and cork manufactures } \\
& \text { 64. Paper and paperboard manufactures } \\
& \text { 65. Textile manufactures } \\
& \text { 67. Iron and steel } \\
& \text { 68. Non-ferrous metals } \\
& \text { 69. Metal manufactures }
\end{aligned}
$$

7. Machinery and transport equipment ${ }^{2}$

$71-716,72,73 \& 74$. Mechanical machinery

$716,75,76$ \& 77 . Electrical machinery

78. Road vehicles

79. Other transport equipment

8. Miscellaneous manufactures ${ }^{2}$

Of which:

84. Clothing
85. Footwear
87 \& 88. Scientific and photographic

9. Other commodities and transactions
BQOY

$\mathrm{BQOZ}$

$B O P A$

$B Q P B$

BOPN

ELBL

BOQI

BQPI

ENDG

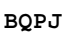

BQPK

CSCE

BQPL

CSCF

BQQA

BQQB

BQQC

BQQD

$B Q Q E$

$B Q Q F$

$\mathrm{BOOG}$

$\mathrm{BQQH}$

BQQI

BQQK

BQQL

BQQM

$B O O N$

BQQO $\begin{array}{rr}144 & 126 \\ 417 & 481 \\ 499 & 543 \\ 2898 & 3665\end{array}$

$24700 \quad 35413$

22756

1944

31874

3539

$\begin{array}{rr}28 & 22 \\ 97 & 82 \\ 150 & 128 \\ 569 & 479\end{array}$

$\begin{array}{rr}20 & 6 \\ 87 & 27 \\ 145 & 50\end{array}$

$588 \quad 187$

$7693 \quad 6195$

$6363 \quad 2044$

$6718 \quad 5401$

$\begin{array}{lrrr}975 & 794 & 5741 & 1837\end{array}$

$90 \quad 142$

$98 \quad 45$

8891

38891

43785

$\begin{array}{rrrrrrrrrrr}7601 & 8389 & 2106 & 2023 & 1884 & 592 & 607 & 563 & 714 & 821^{\dagger} & 991 \\ 2830 & 2983 & 659 & 897 & 629 & 238 & 248 & 179 & 202 & 246^{\dagger} & 220 \\ 1672 & 1837 & 430 & 404 & 410 & 137 & 139 & 134 & 137 & 138^{\dagger} & 140 \\ 14507 & 17228 & 4708 & 5087 & 4994 & 1602 & 1805 & 1566 & 1623 & 1656^{\dagger} & 1653 \\ 3689 & 3945 & 1002 & 1000 & 1007 & 332 & 336 & 324 & 347 & 349^{\dagger} & 353 \\ 4612 & 4859 & 1112 & 1075 & 1102 & 356 & 372 & 373 & 357 & 375^{\dagger} & 367 \\ 29378 & 32394 & 7507 & 6183 & 6096 & 2122 & 2047 & 2045 & 2004 & 2113^{\dagger} & 2063\end{array}$

272

$\begin{array}{ll}2124 & 2335 \\ 2589 & 2591\end{array}$

$561-571$

$\begin{array}{llll}6853 & 1556 & 1290 & 1041\end{array}$

$\begin{array}{llrrr}5778 & 6866 & 1644 & 947 & 956 \\ 4665 & 5045 & 1237 & 1109 & 1088\end{array}$

$\begin{array}{lllllll}82713 & 89189 & 21472 & 19777 & 19176 & 6676\end{array}$

$\begin{array}{llllll}28969 & 32273 & 7922 & 7580 & 7484 & 2618\end{array}$

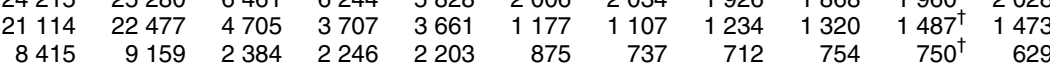

$\begin{array}{lllllllllll}26695 & 28468 & 7238 & 6876 & 6838 & 2340 & 2286 & 2272 & 2280 & 2385^{\dagger} & 2233\end{array}$ $\begin{array}{lrrrrrrrrrrr}\text { CSCN } & 3100 & 3305 & 865 & 860 & 851 & 282 & 286 & 282 & 283 & 285^{\dagger} & 294\end{array}$

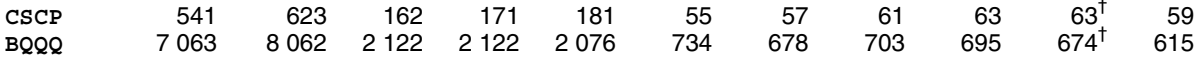

BOOL

1 The numbers on the left hand side of the table refer to the code numbers of 2 Sections 7 and 8 are shown by broad economic category in table G2 of the the Standard International Trade Classification, Revision 3, which was intro- Monthly Review of External Trade Statistics.

duced in January 1988. 
15.7 United Kingdom imports, by commodity'

$£$ million Balance of Payments-consistent basis seasonally adjusted

\begin{tabular}{|c|c|c|c|c|c|c|c|c|c|c|c|c|}
\hline & & 2007 & 2008 & $\begin{array}{r}2008 \\
\text { Q4 }\end{array}$ & $\begin{array}{r}2009 \\
\text { Q1 }\end{array}$ & $\begin{array}{r}2009 \\
\text { Q2 }\end{array}$ & $\begin{array}{r}2009 \\
\text { Mar }\end{array}$ & $\begin{array}{r}2009 \\
\text { Apr }\end{array}$ & $\begin{array}{r}2009 \\
\text { May }\end{array}$ & $\begin{array}{r}2009 \\
\text { Jun }\end{array}$ & $\begin{array}{r}2009 \\
\text { Jul }\end{array}$ & $\begin{array}{r}2009 \\
\text { Aug }\end{array}$ \\
\hline 0. Food and live animals & BQQR & 21324 & 25291 & 6630 & 6604 & 6716 & 2191 & 2294 & 2228 & 2194 & $2132^{\dagger}$ & 21 \\
\hline \multicolumn{13}{|l|}{ Of which: } \\
\hline 01. Meat and meat preparations & BQQS & 3992 & 4618 & 1199 & 1255 & 1249 & 420 & 413 & 412 & 424 & $395^{\dagger}$ & \\
\hline 02. Dairy products and eggs & $\mathrm{BQQT}$ & 1837 & 2281 & 560 & 583 & 590 & 198 & 191 & 201 & 198 & $184^{\dagger}$ & \\
\hline $04 \& 08$. Cereals and animal feeding stuffs & $B Q Q U$ & 2918 & 3814 & 931 & 980 & 1029 & 322 & 350 & 337 & 342 & $342^{\dagger}$ & \\
\hline 1. Beverages and tobacco & BQQW & 5423 & 5831 & 1428 & 1489 & 1507 & 500 & 517 & 492 & 498 & $481^{\dagger}$ & \\
\hline 11. Beverages & EGAT & 3942 & 4318 & 1040 & 1091 & 1098 & 366 & 378 & 352 & 368 & $357^{\dagger}$ & \\
\hline 12. Tobacco & EMAI & 1481 & 1513 & 388 & 398 & 409 & 134 & 139 & 140 & 130 & $124^{\dagger}$ & \\
\hline 2. Crude materials & ENVB & 8663 & 9595 & 2039 & 1633 & 1583 & 558 & 549 & 533 & 501 & $492^{\dagger}$ & \\
\hline
\end{tabular}

Of which:

24. Wood, lumber and cork
25. Pulp and waste paper
26. Textile fibres
28. Metal ores

3. Fuels

33. Petroleum and petroleum products

32, 34 and 35 . Coal, gas and electricity

4. Animal and vegetable oils and fats

5. Chemicals

Of which:

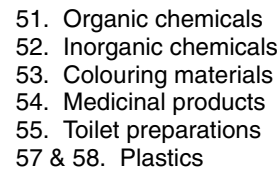

6. Manufactures classified chiefly by material

Of which:

63. Wood and cork manufactures

64. Paper and paperboard manufactures

65. Textile manufactures

67. Iron and steel

68. Non-ferrous metals

69. Metal manufactures

7. Machinery and transport equipment ${ }^{2}$

$71-716,72,73 \& 74$. Mechanical machinery

$716,75,76$ \& 77 . Electrical machinery

78. Road vehicles

79. Other transport equipment

8. Miscellaneous manufactures ${ }^{2}$

Of which:

84. Clothing
85. Footwear
87 \& 88. Scientific and photographic

9. Other commodities and transactions
ENVC

EQAH

EQAP

EHAA

BQAT

ENXO

BPBI

EHAB

ENGA

EHAC

EHAE

CSCR

EHAF

CSCS

EHAG

EHAH

EHAI

EHAJ

EHAK

EHAL

EHAM

EHAN

EHAO

EHAQ

EHAR

EHAS

EHAT

EHAU
1805

503

315

1410
595

595
335

281
15
80

$151 \quad 129$

31928

26787

5141

$48367 \quad 10743$

8747

37802

7682

619

898

1399

347

307

$34645 \quad 37928$

\section{0 \\ 2679}

1164

9943

3448

8460
2756

1228

11049

6219

39792

41910

1733
5248
4084
5958
6230
6563

$\begin{array}{rrrr}1731 & 397 & 368 & 378\end{array}$

$\begin{array}{rrrr}5492 & 1379 & 1358 & 1350 \\ 4073 & 981 & 953 & 931\end{array}$

$\begin{array}{llll}6575 & 1519 & 1029 & 892\end{array}$

$\begin{array}{llll}6419 & 1395 \quad 1447 \quad 1518\end{array}$

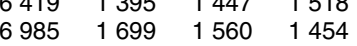

$\begin{array}{llllll}117726 & 121010 & 28537 & 25575 & 25308 & 8289\end{array}$

$\begin{array}{llllll}25776 & 28916 & 7015 & 6321 & 6012 & 1999\end{array}$

$\begin{array}{lllllllllll}36590 & 33924 & 6585 & 5564 & 5837 & 1879 & 1827 & 1879 & 2131 & 2282^{\dagger} & 2346\end{array}$

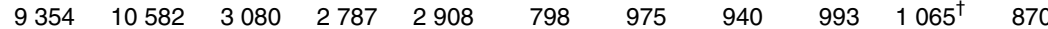

$\begin{array}{lllllllllll}47939 & 50921 & 12926 & 12888 & 12466 & 4081 & 4281 & 4117 & 4068 & 4104^{\dagger} & 4082\end{array}$

1 The numbers on the left hand side of the table refer to the code numbers of 2 Sections 7 and 8 are shown by broad economic category in table G2 of the the Standard International Trade Classification, Revision 3, which was intro- Monthly Review of External Trade Statistics.

duced in January 1988. 


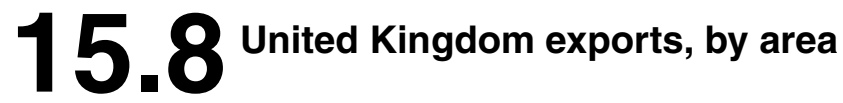

$£$ million Balance of Payments-consistent basis seasonally adjusted

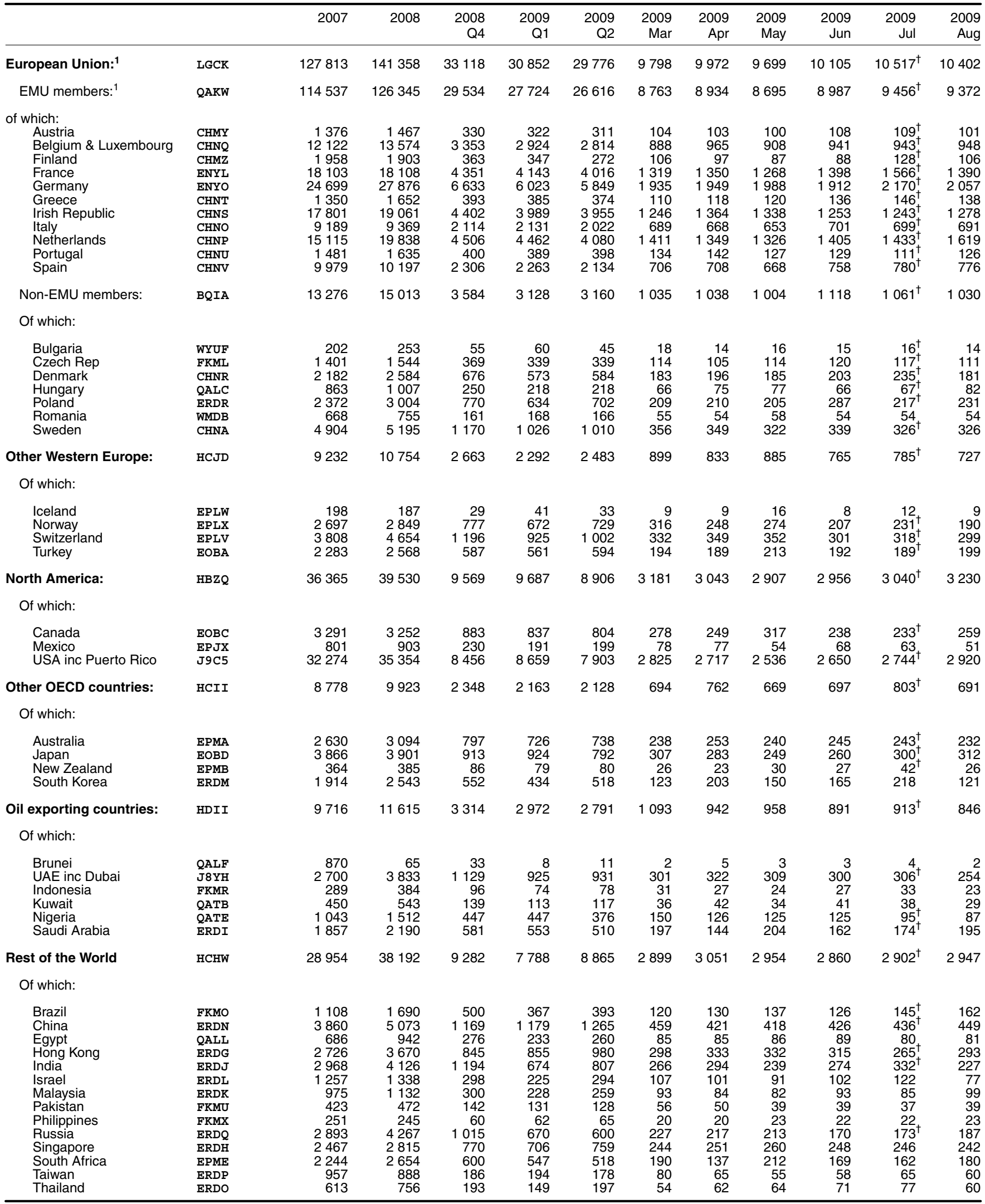

1 Includes; Austria, Belgium, Cyprus, Czech Republic, Denmark, Estonia, Lithuania, Luxembourg, Malta, Netherlands, Poland, Portugal, Romania, SloFinland, France, Germany, Greece, Hungary, Irish Republic, Italy, Latvia, 
$£$ million Balance of Payments-consistent basis seasonally adjusted

\begin{tabular}{|c|c|c|c|c|c|c|c|c|c|c|c|c|}
\hline & & 2007 & 2008 & $\begin{array}{r}2008 \\
\text { Q4 }\end{array}$ & $\begin{array}{r}2009 \\
\text { Q1 }\end{array}$ & $\begin{array}{r}2009 \\
\text { Q2 }\end{array}$ & $\begin{array}{r}2009 \\
\text { Mar }\end{array}$ & $\begin{array}{r}2009 \\
\text { Apr }\end{array}$ & $\begin{array}{r}2009 \\
\text { May }\end{array}$ & $\begin{array}{r}2009 \\
\text { Jun }\end{array}$ & $\begin{array}{r}2009 \\
\text { Jul }\end{array}$ & $\begin{array}{r}2009 \\
\text { Aug }\end{array}$ \\
\hline European Union: ${ }^{1}$ & LGDC & 169799 & 180841 & 41914 & 39117 & 38695 & 12995 & 12984 & 12758 & 12953 & $13060^{\dagger}$ & 13596 \\
\hline EMU members & QAKX & 149719 & 157834 & 36603 & 34477 & 33812 & 11463 & 11446 & 11156 & 11210 & $11286^{\dagger}$ & 11780 \\
\hline $\begin{array}{l}\text { of which: } \\
\text { Austria } \\
\text { Belgium \& Luxembourg } \\
\text { Finland } \\
\text { France } \\
\text { Germany } \\
\text { Greece } \\
\text { Irish Republic } \\
\text { Italy } \\
\text { Netherlands } \\
\text { Portugal } \\
\text { Spain }\end{array}$ & $\begin{array}{l}\text { CHNB } \\
\text { CHNY } \\
\text { CHNC } \\
\text { ENYP } \\
\text { ENYS } \\
\text { CHOB } \\
\text { CHOA } \\
\text { CHNW } \\
\text { CHNX } \\
\text { CHOC } \\
\text { CHOD }\end{array}$ & $\begin{array}{r}2488 \\
15820 \\
2619 \\
21896 \\
44565 \\
640 \\
11338 \\
13316 \\
23079 \\
1506 \\
10489\end{array}$ & $\begin{array}{r}2344 \\
17323 \\
2782 \\
23172 \\
44634 \\
646 \\
12232 \\
14144 \\
25806 \\
1744 \\
10773\end{array}$ & $\begin{array}{r}568 \\
3985 \\
645 \\
5423 \\
10161 \\
141 \\
3014 \\
3272 \\
5892 \\
429 \\
2435\end{array}$ & $\begin{array}{r}569 \\
3664 \\
635 \\
5094 \\
9488 \\
128 \\
3087 \\
2987 \\
5654 \\
343 \\
2290\end{array}$ & $\begin{array}{r}538 \\
3746 \\
618 \\
4986 \\
9336 \\
126 \\
3280 \\
2866 \\
5245 \\
335 \\
2283\end{array}$ & $\begin{array}{r}191 \\
1232 \\
213 \\
1718 \\
3188 \\
40 \\
953 \\
985 \\
1829 \\
114 \\
783\end{array}$ & $\begin{array}{r}179 \\
1189 \\
197 \\
1668 \\
3107 \\
42 \\
1211 \\
973 \\
1813 \\
121 \\
771\end{array}$ & $\begin{array}{r}184 \\
1280 \\
200 \\
1629 \\
3119 \\
41 \\
1050 \\
955 \\
1731 \\
103 \\
751\end{array}$ & $\begin{array}{r}175 \\
1277 \\
221 \\
1689 \\
3110 \\
43 \\
1019 \\
938 \\
1701 \\
111 \\
761\end{array}$ & $\begin{array}{r}180^{\dagger} \\
1362^{\dagger} \\
218^{\dagger} \\
1690^{\dagger} \\
3160^{\dagger} \\
53^{\dagger} \\
866^{\dagger} \\
957^{\dagger} \\
1702^{\dagger} \\
111^{\dagger} \\
829^{\dagger}\end{array}$ & $\begin{array}{r}191 \\
1405 \\
265 \\
1844 \\
3343 \\
42 \\
940 \\
946 \\
1658 \\
109 \\
860\end{array}$ \\
\hline Non-EMU members: ${ }^{1}$ & $B Q I B$ & 20080 & 23007 & 5311 & 4640 & 4883 & 1532 & 1538 & 1602 & 1743 & $1774^{\dagger}$ & 1816 \\
\hline $\begin{array}{l}\text { Of which: } \\
\text { Bulgaria } \\
\text { Czech Rep } \\
\text { Denmark } \\
\text { Hungary } \\
\text { Poland } \\
\text { Romania } \\
\text { Sweden }\end{array}$ & $\begin{array}{l}\text { WYUT } \\
\text { FKMM } \\
\text { CHNZ } \\
\text { QALD } \\
\text { ERED } \\
\text { WMDC } \\
\text { CHND }\end{array}$ & $\begin{array}{r}239 \\
2983 \\
3444 \\
2377 \\
3695 \\
938 \\
5274\end{array}$ & $\begin{array}{r}207 \\
3577 \\
3920 \\
2524 \\
4306 \\
806 \\
6799\end{array}$ & $\begin{array}{r}43 \\
880 \\
941 \\
500 \\
1070 \\
186 \\
1533\end{array}$ & $\begin{array}{r}37 \\
750 \\
776 \\
458 \\
982 \\
186 \\
1264\end{array}$ & $\begin{array}{r}37 \\
784 \\
779 \\
524 \\
1044 \\
176 \\
1332\end{array}$ & $\begin{array}{r}13 \\
253 \\
216 \\
119 \\
333 \\
59 \\
457\end{array}$ & $\begin{array}{r}13 \\
269 \\
249 \\
149 \\
324 \\
59 \\
407\end{array}$ & $\begin{array}{r}12 \\
251 \\
256 \\
159 \\
354 \\
57 \\
447\end{array}$ & $\begin{array}{r}12 \\
264 \\
274 \\
216 \\
366 \\
60 \\
478\end{array}$ & $\begin{array}{r}13 \\
272^{\dagger} \\
262^{\dagger} \\
165^{\dagger} \\
451^{\dagger} \\
61^{\dagger} \\
481^{\dagger}\end{array}$ & $\begin{array}{r}14 \\
311 \\
248 \\
208 \\
384 \\
58 \\
517\end{array}$ \\
\hline $\begin{array}{l}\text { Other Western Europe: } \\
\text { Of which: }\end{array}$ & HBTS & 24359 & 32432 & 7401 & 6734 & 5976 & 2327 & 2154 & 1791 & 2031 & $2182^{\dagger}$ & 2204 \\
\hline $\begin{array}{l}\text { Iceland } \\
\text { Norway } \\
\text { Switzerland } \\
\text { Turkey }\end{array}$ & $\begin{array}{l}\text { EPMW } \\
\text { EPMX } \\
\text { EPMV } \\
\text { EOBU }\end{array}$ & $\begin{array}{r}415 \\
14316 \\
4746 \\
4632\end{array}$ & $\begin{array}{r}457 \\
21606 \\
5256 \\
4874\end{array}$ & $\begin{array}{l}107 \\
4763 \\
1344 \\
1126\end{array}$ & $\begin{array}{rl} & 114 \\
4 & 232 \\
1 & 265 \\
1 & 072\end{array}$ & $\begin{array}{l}123 \\
3399 \\
1275 \\
1124\end{array}$ & $\begin{array}{r}40 \\
1441 \\
418 \\
408\end{array}$ & $\begin{array}{r}34 \\
1257 \\
476 \\
368\end{array}$ & $\begin{array}{r}47 \\
959 \\
389 \\
377\end{array}$ & $\begin{array}{r}42 \\
1183 \\
410 \\
379\end{array}$ & $\begin{array}{r}36 \\
1289^{\dagger} \\
418^{\dagger} \\
424^{\dagger}\end{array}$ & $\begin{array}{r}42 \\
1359 \\
392 \\
382\end{array}$ \\
\hline $\begin{array}{l}\text { North America: } \\
\text { Of which: }\end{array}$ & HCRB & 32472 & 32562 & 8277 & 8512 & 7334 & 2870 & 2700 & 2339 & 2295 & $2327^{\dagger}$ & 2161 \\
\hline $\begin{array}{l}\text { USA inc Puerto Rico } \\
\text { Canada } \\
\text { Mexico }\end{array}$ & $\begin{array}{l}\text { J9C6 } \\
\text { EOBW } \\
\text { EPJY }\end{array}$ & $\begin{array}{r}26095 \\
5793 \\
582\end{array}$ & $\begin{array}{r}25957 \\
5812 \\
793\end{array}$ & $\begin{array}{r}6615 \\
1452 \\
210\end{array}$ & $\begin{array}{r}7030 \\
1295 \\
187\end{array}$ & $\begin{array}{rl}6 & 014 \\
1 & 142 \\
178\end{array}$ & $\begin{array}{r}2345 \\
436 \\
89\end{array}$ & $\begin{array}{r}2186 \\
437 \\
77\end{array}$ & $\begin{array}{r}1859 \\
422 \\
58\end{array}$ & $\begin{array}{r}1969 \\
283 \\
43\end{array}$ & $\begin{array}{c}1921^{\dagger} \\
356^{\dagger} \\
50\end{array}$ & $\begin{array}{r}1784 \\
291 \\
86\end{array}$ \\
\hline $\begin{array}{l}\text { Australia } \\
\text { Japan } \\
\text { New Zealand } \\
\text { South Korea }\end{array}$ & $\begin{array}{l}\text { EPNA } \\
\text { EOBX } \\
\text { EPNB } \\
\text { ERDY }\end{array}$ & $\begin{array}{r}2245 \\
7885 \\
667 \\
3073\end{array}$ & $\begin{array}{r}2385 \\
8535 \\
747 \\
3506\end{array}$ & $\begin{array}{r}761 \\
2125 \\
181 \\
870\end{array}$ & $\begin{array}{r}668 \\
1649 \\
221 \\
782\end{array}$ & $\begin{array}{r}522 \\
1631 \\
209 \\
807\end{array}$ & $\begin{array}{r}136 \\
518 \\
73 \\
244\end{array}$ & $\begin{array}{r}176 \\
518 \\
67 \\
307\end{array}$ & $\begin{array}{r}192 \\
466 \\
68 \\
266\end{array}$ & $\begin{array}{r}154 \\
647 \\
74 \\
234\end{array}$ & $\begin{array}{c}208^{\dagger} \\
594^{\dagger} \\
66^{\dagger} \\
235^{\dagger}\end{array}$ & $\begin{array}{r}154 \\
574 \\
64 \\
221\end{array}$ \\
\hline $\begin{array}{l}\text { Oil exporting countries: } \\
\text { Of which: }\end{array}$ & HCPC & 6387 & 7992 & 1869 & 1774 & 1875 & 660 & 654 & 625 & 596 & $655^{\dagger}$ & 537 \\
\hline $\begin{array}{l}\text { Brunei } \\
\text { UAE inc Dubai } \\
\text { Indonesia } \\
\text { Kuwait } \\
\text { Nigeria } \\
\text { Saudi Arabia }\end{array}$ & $\begin{array}{l}\text { QALG } \\
\text { J8YI } \\
\text { FKMS } \\
\text { QATC } \\
\text { QATF } \\
\text { ERDU }\end{array}$ & $\begin{array}{r}57 \\
1015 \\
925 \\
696 \\
271 \\
821\end{array}$ & $\begin{array}{r}27 \\
933 \\
1184 \\
1090 \\
910 \\
673\end{array}$ & $\begin{array}{r}2 \\
213 \\
333 \\
173 \\
334 \\
148\end{array}$ & $\begin{array}{r}12 \\
298 \\
329 \\
142 \\
102 \\
169\end{array}$ & $\begin{array}{r}18 \\
223 \\
314 \\
212 \\
192 \\
198\end{array}$ & $\begin{array}{r}7 \\
82 \\
108 \\
58 \\
25 \\
90\end{array}$ & $\begin{array}{r}17 \\
78 \\
112 \\
84 \\
4 \\
95\end{array}$ & $\begin{array}{r}1 \\
73 \\
104 \\
49 \\
141 \\
36\end{array}$ & $\begin{array}{l}7 \overline{2} \\
98 \\
79 \\
47 \\
67\end{array}$ & $\begin{array}{l}4 \\
87^{\dagger} \\
97^{\dagger} \\
78^{\dagger} \\
70 \\
30\end{array}$ & $\begin{array}{r}1 \\
78 \\
93 \\
37 \\
3 \\
40\end{array}$ \\
\hline $\begin{array}{l}\text { Rest of the World } \\
\text { Of which: }\end{array}$ & HCIF & 63725 & 75818 & 19268 & 17093 & 17786 & 5231 & 6091 & 5890 & 5805 & $6064^{\dagger}$ & 5572 \\
\hline $\begin{array}{l}\text { Brazil } \\
\text { China } \\
\text { Egypt } \\
\text { Hong Kong } \\
\text { India } \\
\text { Israel } \\
\text { Malaysia } \\
\text { Pakistan } \\
\text { Philippines } \\
\text { Russia } \\
\text { Singapore } \\
\text { South Africa } \\
\text { Taiwan } \\
\text { Thailand }\end{array}$ & $\begin{array}{l}\text { FKMP } \\
\text { ERDZ } \\
\text { QALM } \\
\text { ERDS } \\
\text { ERDV } \\
\text { ERDX } \\
\text { ERDW } \\
\text { FKMV } \\
\text { FKMY } \\
\text { EREC } \\
\text { ERDT } \\
\text { EPNE } \\
\text { EREB } \\
\text { EREA }\end{array}$ & $\begin{array}{r}2061 \\
18734 \\
538 \\
6939 \\
3809 \\
1045 \\
1684 \\
512 \\
717 \\
5248 \\
4247 \\
3060 \\
2418 \\
2012\end{array}$ & $\begin{array}{r}2617 \\
23154 \\
639 \\
8074 \\
4487 \\
1155 \\
1871 \\
630 \\
628 \\
6921 \\
4003 \\
4734 \\
2594 \\
2424\end{array}$ & $\begin{array}{r}653 \\
6322 \\
135 \\
2060 \\
1101 \\
273 \\
482 \\
163 \\
132 \\
1644 \\
1099 \\
968 \\
601 \\
649\end{array}$ & $\begin{array}{r}625 \\
6036 \\
167 \\
1941 \\
1138 \\
270 \\
447 \\
171 \\
110 \\
1270 \\
856 \\
808 \\
558 \\
581\end{array}$ & $\begin{array}{r}669 \\
6116 \\
191 \\
1889 \\
1093 \\
272 \\
421 \\
156 \\
110 \\
1163 \\
914 \\
1070 \\
519 \\
575\end{array}$ & $\begin{array}{r}201 \\
1723 \\
60 \\
621 \\
386 \\
84 \\
153 \\
60 \\
38 \\
464 \\
291 \\
368 \\
175 \\
198\end{array}$ & $\begin{array}{r}265 \\
2127 \\
52 \\
645 \\
361 \\
83 \\
139 \\
51 \\
35 \\
383 \\
339 \\
408 \\
176 \\
185\end{array}$ & $\begin{array}{r}172 \\
1994 \\
65 \\
613 \\
373 \\
92 \\
139 \\
47 \\
37 \\
339 \\
347 \\
335 \\
170 \\
190\end{array}$ & $\begin{array}{r}232 \\
1995 \\
74 \\
631 \\
359 \\
97 \\
143 \\
58 \\
38 \\
441 \\
228 \\
327 \\
173 \\
200\end{array}$ & $\begin{array}{r}219^{\dagger} \\
2025^{\dagger} \\
70^{\dagger} \\
635^{\dagger} \\
393^{\dagger} \\
86^{\dagger} \\
143 \\
56^{\dagger} \\
39^{\dagger} \\
396^{\dagger} \\
237^{\dagger} \\
432^{\dagger} \\
182^{\dagger} \\
183^{\dagger}\end{array}$ & $\begin{array}{r}186 \\
2023 \\
33 \\
632 \\
391 \\
83 \\
131 \\
64 \\
31 \\
296 \\
244 \\
145 \\
185 \\
188\end{array}$ \\
\hline
\end{tabular}

1 Includes; Austria, Belgium, Bulgaria, Cyprus, Czech Republic, Denmark,

Estonia, Finland, France, Germany, Greece, Hungary, Irish Republic, Italy,

Source: Office for National Statistics: 01633456294

Latvia, Lithuania, Luxembourg, Malta, Netherlands, Poland, Portugal, Ro-

mania, Slovakia, Slovenia, Spain, and Sweden. 